\title{
Small angles X-ray diffraction and Mössbauer characterization of annealed $\mathrm{Tb} / \mathrm{Fe}$ multilayer
}

\author{
O M LEMINE \\ Physics Department, College of Sciences, Imam University, Riyadh, Saudi Arabia
}

MS received 17 November 2007; revised 11 January 2011

\begin{abstract}
The effect of thermal annealing on the structure and magnetic properties of crystalline Tb/Fe multilayers has been studied using conversion electron Mössbauer spectrometry and small-angle X-ray diffraction. The growth of $\mathrm{Tb}-\mathrm{Fe}$ amorphous alloy from the interface is observed with increasing annealing temperature. After annealing at $873 \mathrm{~K}$, a clear total mixing of the multilayers by interdiffusion has been evidenced. The results are compared with the effect of ion irradiation in the same system.
\end{abstract}

Keywords. Mössbauer spectrometry; annealing; magnetism; Tb/Fe multilayers.

\section{Introduction}

In the last decades iron/rare earth (Fe/RE) multilayers have been extensively studied for their structural and magnetic properties. These multilayers showing properties like perpendicular magnetic anisotropy (PMA) in system like Fe-Tb multilayers is significantly affected by their interfacial structure (Scholz et al 1992; Tappert et al 1996, 1999; Lanchava and Hoffmann 1999). For the Tb/Fe multilayers, both demixing and mixing at the interface is induced by ion irradiation (Teillet et al 1997; Gupta et al 1998). On the other hand, in a study of the effect of annealing in $\mathrm{Fe}-\mathrm{Tb}$ multilayers with the structure of the Fe layer being amorphous, initially a demixing is observed followed by intermixing at higher temperatures (Findiki et al 1997). However, in system with amorphous Fe layers the results are ambiguous, as it is difficult to distinguish between intermixed layers at the interface and the bulk of the amorphous Fe layers. In this paper, we report the annealing effect on $\mathrm{Tb}-\mathrm{Fe}$ multilayers having crystalline bcc Fe layers.

\section{Experimental}

Multilayered films of nominal $[\mathrm{Tb}(35 \AA) / \mathrm{Fe}(45 \AA)] \times 30$ thicknesses were grown by evaporation and alternate condensation of each element in a high vacuum chamber. The pressure during the evaporation was kept below $10^{-8}$ Torr. The substrates were float glass plates and Kapton foils, and were kept at a fixed $T_{\mathrm{s}}$ temperature of $90 \mathrm{~K}$ during the deposition process. To prevent oxidation upon exposure to atmo-

(leminej@yahoo.com) sphere, the sample was over-coated with $140 \AA$ amorphous silicon. Thermal annealing $\left(T_{\mathrm{a}}\right)$ was done at $573 \mathrm{~K}, 773 \mathrm{~K}$ and $873 \mathrm{~K}$ for $3 \mathrm{~h}$ in an evacuated quartz tube within an accuracy of $\pm 1^{\circ} \mathrm{C}$. Small angle X-ray diffraction (SAXRD) and conversion electron Mössbauer spectrometry (CEMS) were used to characterize the multilayer before and after

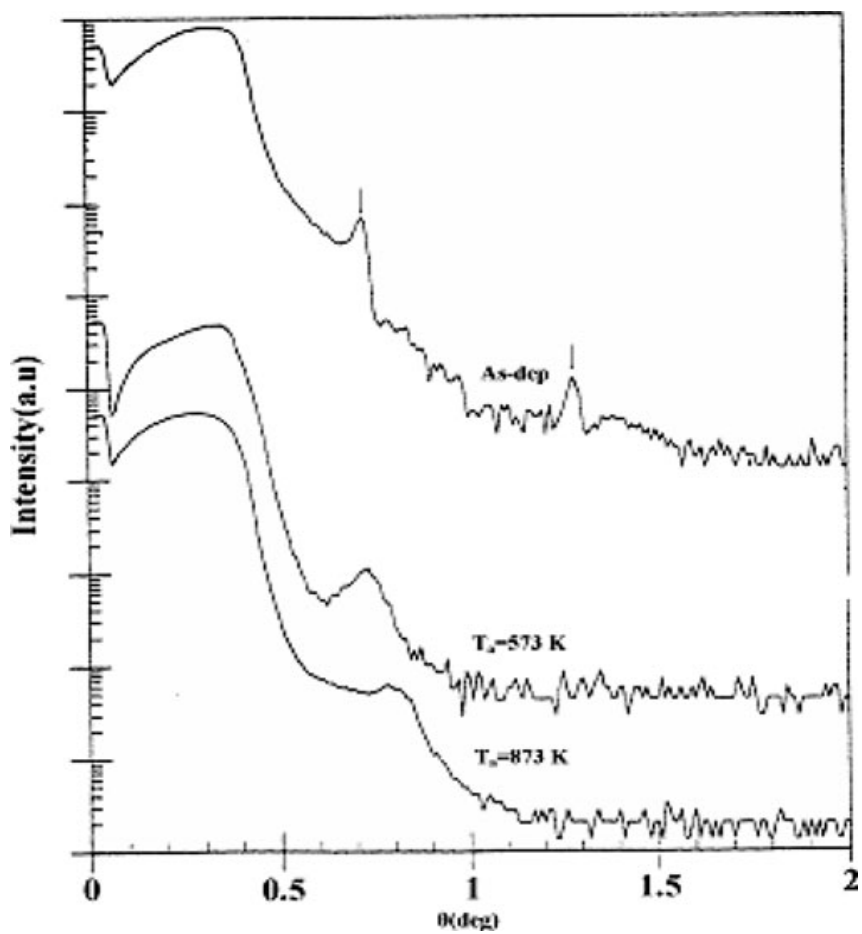

Figure 1. Low angle $\mathrm{X}$-ray diffraction pattern for as-deposited and annealed $[\mathrm{Tb}(35 \AA) / \mathrm{Fe}(45 \AA)] \times 30$ multilayers. 
annealing. For the SAXRD measurements, we used the K $\alpha$ line of cobalt $(\lambda=1.7889 \AA) .{ }^{57} \mathrm{Fe}$ CEMS spectra were recorded at room temperature using a flowing gas $(95 \% \mathrm{He}-$ $5 \% \mathrm{CH} 4)$ proportional counter and $10 \mathrm{mCi}{ }^{57} \mathrm{Co}$ in a $\mathrm{Rh}$ matrix. The spectra were fitted by means of two components viz. a Zeeman pattern with six Lorentzian lines characteristic of standard $b c c$ iron, and a minor magnetically split broad background with a hyperfine field distribution attributed to iron atoms involved in the $\mathrm{Tb} / \mathrm{Fe}$ interface. Further the relative intensity, $X$, of the second and fifth lines in the spectrum, usually expressed as $3: X: 1 ; 1: X: 3$, are related to angle $\theta$ made by the iron magnetic moments and the incident $\gamma$ beam through $X=4 \sin ^{2} \theta / 1+\cos ^{2} \theta$. As the $\gamma$ beam falls perpendicular onto the sample, $X=4$ and $X=0$ imply that the iron moments are respectively parallel $\left(\theta=90^{\circ}\right)$ and perpendicular $\left(\theta=0^{\circ}\right)$ to the sample plane.

\section{Results and discussion}

Figure 1 gives the low angle $\mathrm{X}$-ray pattern indicating two Bragg peaks characteristic of the multilayer period $\Lambda$. The angular positions $\theta 1=0.72^{\circ}$ and $\theta 2=1.29^{\circ}$ lead to $\Lambda=84 \AA$ in accordance with the nominal period. The CEMS spectrum for untreated sample is displayed in figure 2(a) (left). This spectrum shows two components. A principal component of relative abundance $69 \%$ adjusted with six lines. This component represents the bulk $\alpha$-Fe in
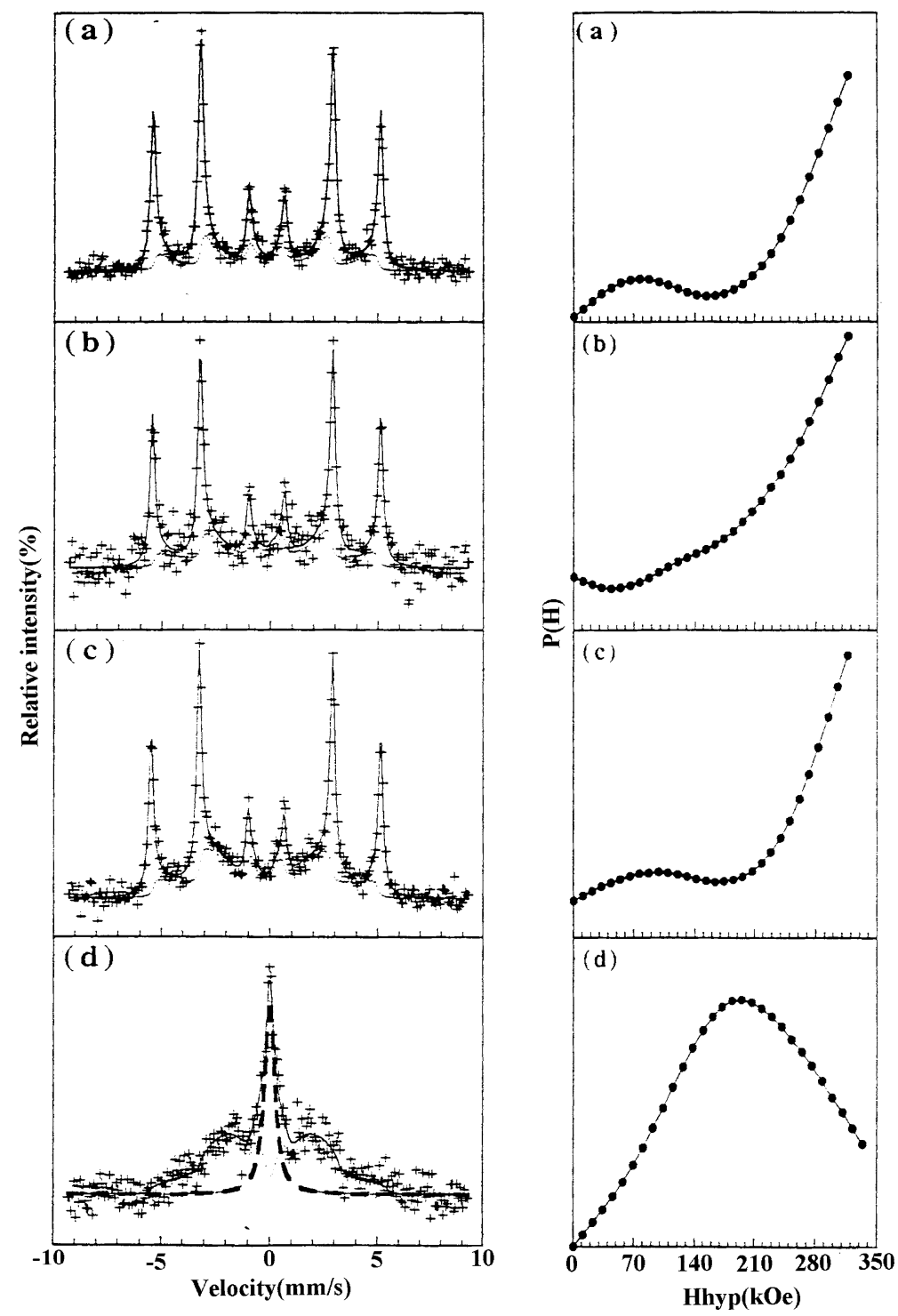

Figure 2. (Left): CEMS spectra corresponding to [Tb $(35 \AA) / \mathrm{Fe}(45 \AA)] \times 30$ multilayers at different annealing temperatures: (a) $300 \mathrm{~K}$, (b) $573 \mathrm{~K}$, (c) $773 \mathrm{~K}$, and (d) $873 \mathrm{~K}$. (Right): hyperfine field distributions. 
Table 1. Results of computing fitting of $\mathrm{Tb}[(35 \AA) / \mathrm{Fe}(45 \AA)] \times$ 30 multilayers of as-deposited and annealed samples.

\begin{tabular}{lrrrr}
\hline $\mathrm{Ta}(\mathrm{K})$ & 300 & 573 & 773 & 873 \\
$\alpha$-Fe $(\%)$ & 69 & 61 & 48 & 0 \\
Interface $(\%)$ & 31 & 39 & 52 & 70 \\
$\mathrm{~Tb}_{0.25} \mathrm{Fe}_{0.75}(\%)$ & 0 & 0 & 0 & 30 \\
$\left\langle B_{\mathrm{hf}}(T)\right\rangle^{*}$ & $22 \cdot 9$ & 22 & 22 & $19 \cdot 6$ \\
\hline
\end{tabular}

$*\left\langle B_{\mathrm{hf}}(T)\right\rangle$ : The average value of the field distribution corresponding to iron atoms involved in the $\mathrm{Tb} / \mathrm{Fe}$ interface.

a layer not disturbed by the presence of terbium. The minor component with large lines is analysed using the histogram method of combined hyperfine field and isomer shift distributions (figure 2(a) (right)). This component is attributed to iron atoms involved in the $\mathrm{Tb} / \mathrm{Fe}$ interface. The relative abundance is $31 \%$. This means that iron atoms in $14 \AA$ out of the $45 \AA$ iron layer sense a depleted hyperfine field due to the terbium neighbourhood.

The spectra of X-ray diffraction of multilayer annealing with $573 \mathrm{~K}$ and $873 \mathrm{~K}$ (figure 1) show that the effect of annealing is to increase roughness (disappearance of the 2nd peak $\theta(2)=1.29^{\circ}$ ) and to shift the first peak to the larger angles $\theta(1)=0.72^{\circ}$ at $573 \mathrm{~K}$ and $\theta(1)=0.78^{\circ}$ at $873 \mathrm{~K}$. There is increase of the interface and decrease of the modulation $\Lambda$ (it passes from $84 \AA$ for the as-deposited sample to $71 \AA$ and $65 \AA$ for successive annealing at $573 \mathrm{~K}$ and $873 \mathrm{~K}$ ). This decrease of the modulation (the thickness $(\mathrm{tTb}+\mathrm{tFe})$ becomes smaller) and increase of interface is explained by the interdiffusion between iron and terbium. The interfaces become rough. The CEMS spectra of the annealed samples at different temperatures are displayed in figures 2(b-d) (right).
Table 1 gives the results of the analysis of CEMS spectra of the annealed samples. With an increase in annealing temperature of $\mathrm{Tb}-\mathrm{Fe}$ multilayers, three observations are made from the fitting of the CEMS spectra: (i) for all Tb/Fe multilayers the increase of the fraction of iron atoms involved in the $\mathrm{Tb} / \mathrm{Fe}$ interface is accompanied by the decrease of $\alpha$-Fe atoms (figure 3 ) with annealing temperature, (ii) for annealing temperatures below $873 \mathrm{~K}$, there remains a nonmixed $\alpha$-Fe and (iii) the significant changes in CEMS spectra which correspond to a mixing caused by annealing are already detected at $\mathrm{Ta}=873 \mathrm{~K}$.

The decrease of the fraction of iron atoms involved in the $\mathrm{Tb} / \mathrm{Fe}$ interface (figure 3 ) is explained by the inter-diffusion between iron and terbium. These results are confirmed by $\mathrm{X}$-ray diffraction (figure 1). In addition, a decrease in hyperfine field $<\mathrm{Bhf}>$ corresponding to the $\mathrm{Tb} / \mathrm{Fe}$ interface is seen (figure 4) which may be due to the diffusion between $\mathrm{Fe}$ and $\mathrm{Tb}$ atoms at the interface. After annealing at $873 \mathrm{~K}$, the hyperfine distribution of the interfacial component is a typical magnetic amorphous alloy. Moreover, the spectrum presents a central part adjusted with broad Lorentzian, representative of an almost paramagnetic amorphous alloy. A comparison of our results with those reported by Kuzmak et al (1995) regarding the mean field found for the $\mathrm{Tb}_{x} \mathrm{Fe}_{1-x}$ homogeneous alloy suggests that the sample annealed at $873 \mathrm{~K}$ is composed of an alloy sequence $\mathrm{Tb}_{0.5} \mathrm{Fe}_{0.5}$ and $\mathrm{Tb}_{0.25}$ $\mathrm{Fe}_{0.75}$. Similar results were also observed by Sajieddine et al (1996). A comparison of the present work with the earlier one (Paul and Gupta 2001) shows that the main effect of annealing in their work is demixing at the interfaces attributed to a positive heat of mixing between $\mathrm{Fe}$ and $\mathrm{Tb}$. This difference between our results may be explained by the range of annealing temperatures used. On the other

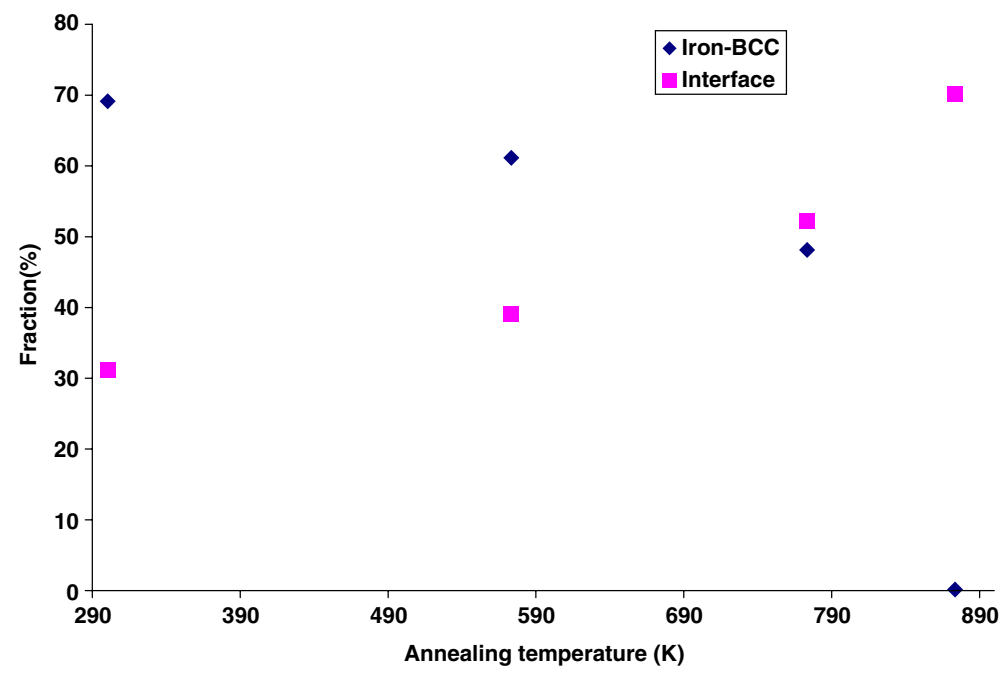

Figure 3. Evolution of the relative fraction of particular component of CEMS spectra versus $T_{\mathrm{a}}$ for the $[\mathrm{Tb}(35 \AA) / \mathrm{Fe}(45 \AA)] \times 30$ multilayers. 


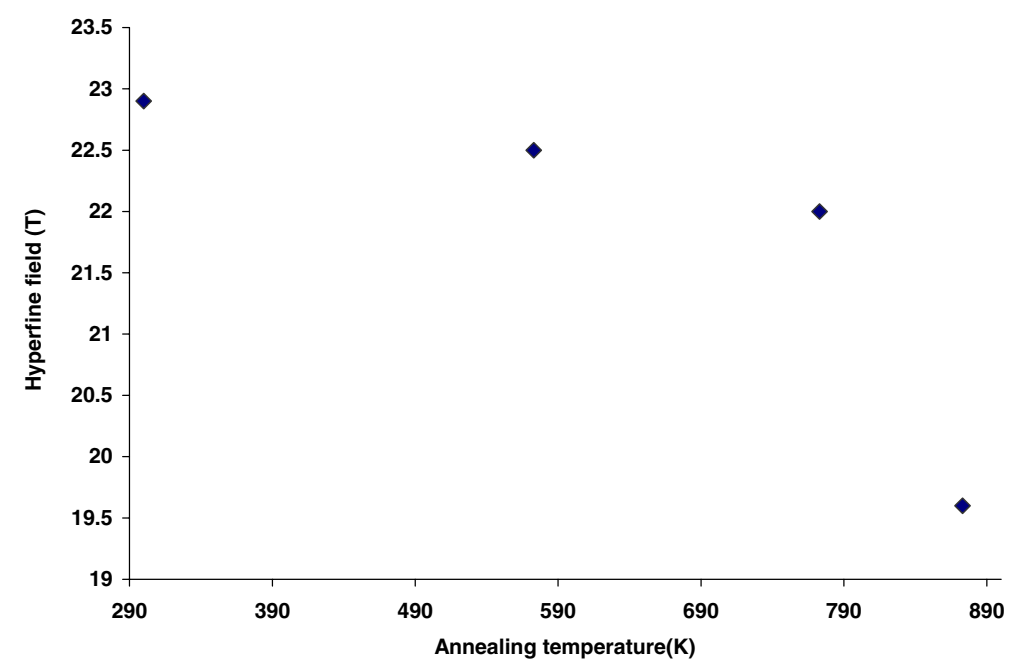

Figure 4. Evolution of the hyperfine field corresponding to the iron atoms involved in the $\mathrm{Tb} / \mathrm{Fe}$ interface versus $T_{\mathrm{a}}$.

hand, in an earlier work (Lemine et al 2006), we have also studied the effect of ion irradiations on $\mathrm{Tb} / \mathrm{Fe}$ multilayers. Specimens of composition similar to the present one were irradiated with $360 \mathrm{keV}$ Ar ions. The mixing of layers $\mathrm{Tb} / \mathrm{Fe}$ is clearly observed and the interface exhibits magnetic properties of amorphous alloys but the mixing efficiency saturates at high fluences and it still remains a crystallized Fe part.

\section{Conclusions}

The thermal annealing effect on magnetic properties of vapor-deposited $\mathrm{Tb} / \mathrm{Fe}$ multilayers was seen by means of conversion electron Mössbauer spectrometry and small angle $\mathrm{X}$-ray diffraction. The growth of $\mathrm{Tb}-\mathrm{Fe}$ amorphous alloy from the interface is observed with increasing annealing temperatures. After annealing at $873 \mathrm{~K}$, a clear mixing of the multilayers by interdiffusion was seen. The mixing is inhomogenous and the sample is composed of an alloy sequence rich in iron and terbium.

\section{References}

Findiki A, Juraszek J, Teillet J, Richomme F and Lebertois J P 1997 J. Magn. Magn. Mater. 165405

Gupta A, Paul A, Gupta R and Principi G 1998 J. Phys., Condens. Matter 109669

Kuzmak O, Pogorily A and Shevchenko V 1995 J. Magn. Magn. Mater. 14836

Lanchava B and Hoffmann H 1999 J. Magn. Magn. Mater. 192403

Lemine O M, Jaouen Ch, Sajieddine M and Bauer Ph 2006 Physica B: Condens. Matter 382266

Paul A and Gupta A 2001 J. Alloy Compd 326246

Sajieddine M, Bauer Ph, Bruson A and Marchal G 1996 Solid State Commun. 99965

Scholz B, Brand R A and Keune W 1992 J. Magn. Magn. Mater 1041089

Tappert J, Keune W, Brand R A, Vulliet P, Sanchez J-P and Shinjo T 1996 J. Appl. Phys. 804503

Tappert J, Neumann S, Brand R A, Keune W, Klose F and Maletta H 1999 Europhys. Lett. 46238

Teillet J, Richomme F, Findiki A and Toulemonde M 1997 Phys. Rev. B55 11560 